\title{
Exploring boar sperm sialylation during capacitation using boronic acid-functionalized beads
}

\author{
Yuanxian Wang ${ }^{1}$, Yihua Gu², ${ }^{2}$, Huihui Gao ${ }^{1}$, Yao Gao ${ }^{1}$, Jianhang Shao ${ }^{1}$, Weijun Pang ${ }^{1}$ and \\ Wuzi Dong ${ }^{1}$ \\ ${ }^{1}$ College of Animal Science and Technology, Northwest A\&F University, Yangling, China, ${ }^{2}$ NPFPC Key Laboratory of \\ Contraceptives and Devices, Shanghai Institute of Planned Parenthood Research (SIPPR), Shanghai, China and \\ ${ }^{3}$ Institutes of Reproduction and Development, Fudan University, Shanghai, China \\ Correspondence should be addressed to W Dong; Email: dongwuzi@nwsuaf.edu.cn
}

\begin{abstract}
Sialic acid (SA), which usually occupies the terminal position of oligosaccharide chains in mammalian spermatozoa, has important functions in fertilization. Compared with other methods, such as lectin probing, boronic acid could recognize and bind SA with a higher affinity and specificity at pH 6.9. In this study, two boronic acid carriers, 3-aminophenylboronic acid-labeled fluorescent latex (CML-APBA) and magnetic beads (CMM-APBA were applied to explore surface sialylation profile and sialoglycoproteins of the boar sperm. There are three binding sections of CML-APBA on the head of ejaculated sperm: acrosomal region, equatorial segment and the head posterior, which are the major regions undergoing sialylation. After capacitation in vitro, two major binding patterns of CML-APBA exists on sperm head. On some spermatozoa, sialylation exists on the equatorial segment and the posterior head, whilst on other spermatozoa, sialylation occurs on the acrosomal region and equatorial segment. Flow cytometry analysis suggested that the level of sialylation on boar sperm membrane decreases after capacitation. Furthermore, using CMM-APBA, we pulled down sialylated proteins from spermatozoa. Among them, two decapacitation factors associating on sperm surface, AWN and PSP-1, were identified. The levels of the two proteins reduced during capacitation, which might contribute to the decrease of sialylation on boar sperm surface.
\end{abstract}

Reproduction (2018) 155 25-36

\section{Introduction}

Spermatozoa take a long journey to undergo a series of changes before one of them fertilizes the oocyte (Fraser 1998). Change on sperm surface carbohydrate profile is one of the key molecular events in the procedure of fertilization. Sialic acid (SA) is the outermost monosaccharide, which caps the majority of glycans at the sperm membrane surface in the epididymis (Young et al. 1986). As an important event during sperm epididymal maturation, accumulation of SA on sperm has been demonstrated in the ram (Holt 1980), bull (Holt 1980), mouse (Ma et al. 2016) and rat (Toowicharanont \& Chulavatnatol 1983) during transit through the epididymis. These studies suggested that epididymal epithelium could synthesize and secrete materials for sialylation of sperm surface or carriers with SA for accumulation on sperm surface. Sialylation changes the physiochemical properties of mammalian cell surface and participate in many physiological functions (Tiralongo \& Martinez-Duncker 2013). For ejaculated spermatozoa, sialylation provide a negatively charged surface, which is a key factor for transit through the cervix (Ozerlat 2011, Tollner et al. 2011). Capacitation is another process of structural and biochemical changes for previously ejaculated spermatozoa to reach oocytes (Aitken \& Nixon 2013). One of the significant changes has been observed that surfaces of mouse and human sperms are desialylated by the presence of sialidase during capacitation, which may unmask certain sperm surface antigens for zona pellucida (ZP) recognition and fertilization (Ma et al. 2012).

SA is a large family of nine-carbon backbone aminosugar that usually occupies the terminal position of oligosaccharide chains in a variety of glycoconjugates (Schauer 1982). The two most common SAs found in mammals are $\mathrm{N}$-acetylneuraminic acid (Neu5Ac) and its derivative $\mathrm{N}$-glycolylneuraminic acid (Neu5Gc) (Rohrer et al. 1998). The assessment of sialylation distribution on sperm surface and quantification of sialylated glycoproteins of sperm might contribute to a better understanding of the function of sialylation in sperm biology. Lectins are capable of recognizing and binding to glycosyl groups with various degrees of specificities. Usually, fluorescence-labeled lectins, such as wheat germ agglutinin (WGA), are applied to research SA location on sperm surface (Magargee et al. 1988, 
Jimenez et al. 2003, Gomez-Torres et al. 2012). However, nonspecific binding has also been reported (Nagata \& Burger 1974). Boronate affinity-functionalized materials have drawn increasing attention due to their capability to selectively react to different glycosyl groups (Lu et al. 2013). Boronic acids can covalently react with cisdiols in the oligosaccharide chains of glycoproteins to form five- or six-membered cyclic esters (Wang et al. 2013). When the reaction $\mathrm{pH}$ is higher than the $\mathrm{p} K_{\mathrm{a}}$ of the boronic acid by one $\mathrm{pH}$ unit or more, the boronate affinity preferentially binds to glycoproteins containing neutral sugars and excludes SA due to electrostatic repulsion. When the binding $\mathrm{pH}<$ the $\mathrm{p} K_{\mathrm{a}}$ by one $\mathrm{pH}$ unit or more, the boronate specifically binds to sialylated glycoproteins due to the exceptional binding affinity of the boronic acid toward SA residues (Lu et al. 2013). This unique chemical property makes the boronic acid group attractive for many applications of recognition, separation and enrichment of glycoproteins. There are many boronic acid-functionalized monolith materials for selective recognition, such as quantum dots (Liu et al. 2011, Huang et al. 2014, Kur-Kowalska et al. 2014, Cao et al. 2015), magnetic beads (Lee et al. 2005, Lin et al. 2011) and so on.

In the present report, bearing boronate groups, two tools were employed for studies of surface sialylation and sialylated proteins of boar sperm. Carboxylatemodified latex-FITC beads, conjugated with 3-aminophenylboronic acid (CML-APBA probe), were used to reveal sialylation profile and profile change on sperm surface during capacitation in vitro. Carboxylatemodified magnetic beads (CMM) conjugated with 3-aminophenylboronic acid (CMM-APBA beads) were used to enrich sialylated proteins of boar spermatozoa. Of them, two sperm surface proteins AWN and PSP-1 were identified. Our study suggested that dissociation of these two sialylated proteins from spermatozoa may also contribute to whole level change of surface sialylation of boar spermatozoa during capacitation in vitro.

\section{Materials and methods}

\section{Ethics}

All breeding pigs were fed in accordance with the Guide for Veterinary Ethical Committee of the Ministry of Agriculture of China and all experimental procedures approved by the Bioethics Committee of Northwest A\&F University.

\section{Preparations of CML-APBA and CMM-APBA}

Schematic representation of the preparation and working principle of CML-APBA or CMM-APBA is shown in Fig. 1.
A<smiles>O=C(O)[CH]c1cccc(B(O)NC(=O)C2CCCCCC2)c1</smiles>

B

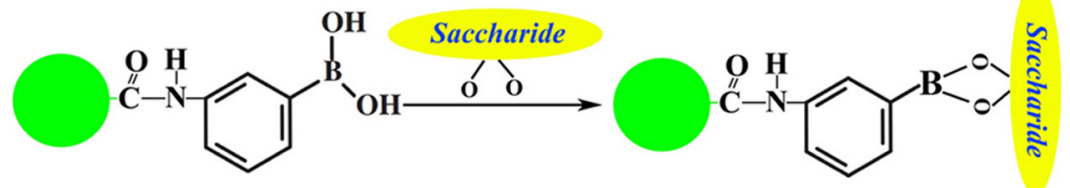

B'

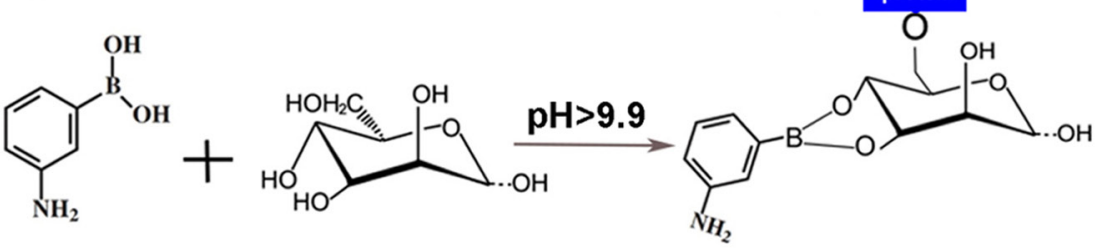

B”'

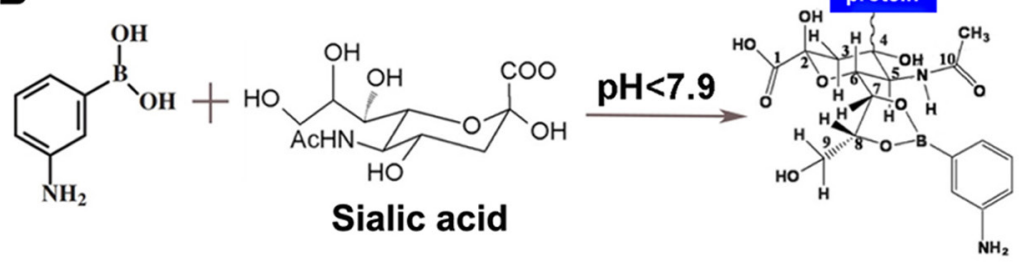

Figure 1 Schematic illustration of synthesis and working principle of CML-APBA or CMM-APBA. (A) Conjugation of carboxylicacid-functionalized beads with 3-aminophenylboronic acid (APBA) by the EDAC-catalyzed reaction. (B) The reaction of APBA with diols in an aqueous solution. $\left(\mathrm{B}^{\prime}\right)$ When binding at a $\mathrm{pH}>9.9$, 3 -aminophenylboronicacid binds with the neutral sugars. ( $\left.\mathrm{B}^{\prime \prime}\right)$ When binding at a $\mathrm{pH}$ $<7.9,3$-aminophenylboronic acid specially binds with sialic acid. 
CML-APBA beads were prepared according to previous reports (Liu et al. 2011, Wang et al. 2013). Briefly, $30 \mu \mathrm{g}$ carboxylatemodified latex beads (CML) (Cat\# L5155, Sigma) were mixed with $1 \mathrm{mg} / \mathrm{mL}$ 3-aminophenylboronic acid (APBA, Sigma) dispersed in a $100 \mathrm{mmol} / \mathrm{L}$ MES buffer solution $(\mathrm{pH} \mathrm{5.5)}$ and gently stirred for $15 \mathrm{~min}$. After that, $10 \mathrm{mg} / \mathrm{mL} \mathrm{N}$-ethyl-NO(3-dimethylaminopropyl)carbodiimide hydrochloride (EDAC, Sigma) was added into the above mixture to start the EDACcatalyzed coupling between APBA and beads. The reaction was carried out for about $2 \mathrm{~h}$ at room temperature. The conjugation mixture was ultrafiltrated (YM-100, Millipore) at $8000 \mathrm{~g}$ for 5 min to remove free APBA and EDAC. After resuspension in phosphate-buffered saline (PBS, $\mathrm{pH}$ 7.4) and one more ultrafiltration, the CML-APBA beads were resuspened in PBS.

Using the same strategy, CMM-APBA beads were prepared. Carboxylate-modified magnetic beads (CMM) (HRCZ04N100) were purchased from Huier (Luoyang, Henan, China). Briefly, the conjugation was carried out for about $2 \mathrm{~h}$ at room temperature. The supernatant containing free APBA AND EADC was removed after CMM beads were bound to the wall of the tube using MagRack6 (GE Healthcare Life Sciences). After triple washing with PBS (pH 7.4), CMM-APBA beads were resuspended in PBS.

The concentration of APBA in the supernatant was measured by matching its absorption maximum at $295 \mathrm{~nm}$ to a predetermined standard curve, and the results were used to calculate the quantity of APBA bound to CML or CMM beads.

\section{Semen collection and sperm treatment}

Semen samples from 10 sexually mature and healthy Guanzhong black boars (2.5-3 years) from the experimental farm of Northwest A\&F University (Yangling, Shaanxi Province, China) were used in this study. Individual ejaculates were collected into a prewarmed tube twice a week by the gloved hand technique before transportation to the laboratory. Each sample was assessed for sperm concentration using a calibrated spectrophotometer and the percentage of motility was evaluated at $37^{\circ} \mathrm{C}$ under phase-contrast microscope. Only the qualified semen samples (more than $10^{7}$ spermatozoa $/ \mathrm{mL}$ and $80 \%$ motility) were selected and used for this study. In the laboratory, the semen samples were centrifuged $(800 \mathrm{~g}$, $10 \mathrm{~min}$ at $17^{\circ} \mathrm{C}$ ) to separate sperm and seminal plasma. The sperm pellets were wished twice with the basic diluents (BTS), which composed of $27.5 \mathrm{~g}$ of glucose, $2.35 \mathrm{~g}$ of EDTA, $6.9 \mathrm{~g}$ of sodium citrate, $2.9 \mathrm{~g}$ of citric acid, $1.0 \mathrm{~g}$ of $\mathrm{NaHCO}_{3}$ and $5.65 \mathrm{~g}$ of Tris for $1000 \mathrm{~mL}$ of deionized water (Estienne et al. 2007). The sperm $\left(5 \times 10^{7}\right.$ spermatozoa $\left./ \mathrm{mL}\right)$ was resuspended in the basic diluent. Those swim-up spermatozoa were collected for the following experiments.

\section{Neuraminidase treatment}

These swim-up spermatozoa in the basic diluent were incubated with neuraminidase (Sigma, N-2876) $(1 \mathrm{IU} / \mathrm{mL}$ in PBS) for $30 \mathrm{~min}$ at $37^{\circ} \mathrm{C}$. Then, the spermatozoa were washed twice to remove neuraminidase. Sperm samples incubated without neuraminidase, as control groups, went through the same procedure.

\section{Capacitation}

Spermatozoa were centrifuged $\left(800 \mathrm{~g}, 10 \mathrm{~min}\right.$ at $\left.17^{\circ} \mathrm{C}\right)$ and the pellets were resuspended in capacitation medium (CM) $\left(95 \mathrm{mmol} / \mathrm{L} \mathrm{NaCl}, 4.8 \mathrm{mmol} / \mathrm{L} \mathrm{KCl}, 2 \mathrm{mmol} / \mathrm{L} \mathrm{CaCl}_{2}, 25 \mathrm{mmol} / \mathrm{L}\right.$ $\mathrm{NaHCO}_{3}, 1.2 \mathrm{mmol} / \mathrm{L} \mathrm{KH}_{2} \mathrm{PO}_{4}, 5.56 \mathrm{mmol} / \mathrm{L}$ glucose, $1 \mathrm{mmol} / \mathrm{L}$ sodium pyruvate $0.6 \% \mathrm{BSA}, \mathrm{pH} 6.9$ ) to a concentration of $5 \times 10^{7} \mathrm{spermatozoa} / \mathrm{mL}$. And then the sperm in extender was incubated for $3 \mathrm{~h}$ at $37^{\circ} \mathrm{C}$ for in vitro capacitation in a humidified incubator with $5 \% \mathrm{CO}_{2}$ (Kumaresan et al. 2014). The control sperm pellets were resuspended in BTS. Then, those swim-up spermatozoa were collected for experiments.

\section{Chlortetracycline (CTC) staining}

The CTC (Anyan, Shanghai, China) staining procedure was essentially carried out as described (Wang et al. 1995). After the capacitation, those swim-up spermatozoa were selected and the capacitation medium or BTS was removed $(800 \mathrm{~g}$, $5 \mathrm{~min}$ at $37^{\circ} \mathrm{C}$ ). Sperm were resuspended in PBS and mixed with equal volume $(45 / 45 \mu \mathrm{L})$ of CTC solution $(750 \mu \mathrm{mol} / \mathrm{L} \mathrm{CTC} \mathrm{in}$ $130 \mathrm{mmol} / \mathrm{L} \mathrm{NaCl}, 5 \mathrm{mmol} / \mathrm{L}$ cysteine, $20 \mathrm{mmol} / \mathrm{L}$ Tris- $\mathrm{HCl}, \mathrm{pH}$ 7.8) and incubated for $30 \mathrm{~min}$ at $37^{\circ} \mathrm{C}$. Cells were then fixed by $8 \mu \mathrm{L}$ of $12.5 \%$ paraformaldehyde in $0.5 \mathrm{mmol} / \mathrm{L}$ Tris- $\mathrm{HCl}$ (pH 6.9) for $10 \mathrm{~min} .10 \mu \mathrm{L}$ of sperm suspension was delivered onto a clean glass slide and covered with a clean coverslip. Then, 300 spermatozoa per slide were observed using a fluorescence microscope equipped with phase-contrast and green epifluorescent optics (Nikon) at $200 \times$ magnification. To avoid evaporation and CTC fading, slides were kept in a wet chamber until the evaluation was carried out.

\section{Microscopy and flow cytometry analyses of spermatozoa bound with CML-APBA probe}

After the capacitation, the swim-up sperm was washed twice in BTS ( $\mathrm{pH}$ 6.9). Sperm sample of each group was incubated with CML-APBA. The protocol was as follows: The CMLAPBA probe was diluted 40 times in PBS ( $\mathrm{pH}$ 6.9). $100 \mu \mathrm{L}$ sperm suspension $\left(5 \times 10^{7}\right.$ spermatozoa $\left./ \mathrm{mL}\right)$ was incubated with $25 \mu \mathrm{L} C M L-A P B A$ probe for $40 \mathrm{~min}$ in the $\mathrm{BTS} \mathrm{pH}=6.9$ at $37^{\circ} \mathrm{C}$. And then the sperm was washed three times (at $800 \mathrm{~g}, 3 \mathrm{~min}$ at $37^{\circ} \mathrm{C}$ ) with PBS ( $\mathrm{pH} \mathrm{6.9)}$ for removing the uncombined probes, the sperm pellets were resuspended in PBS for flow cytometer analysis (FACSCalibur, BD Biosciences, NJ, USA). In the meantime, $5 \mu \mathrm{L}$ sperm suspension was added on clean slip and covered with a clean coverslip. Green fluorescence was observed under microscope equipped with phase-contrast and epifluorescent optics (Nikon) at 400× magnification. Control group of semen were incubated only with CML beads according to the above same protocol, respectively.

\section{Fluorescein isothiocyanate-conjugated wheat germ agglutinin (WGA-FITC) staining of spermatozoa}

After the capacitation process, WGA-FITC (Sigma) was used to label uncapacitated and capacitated sperm cells. The swim-up sperm was divided into two groups. One was treated 
with neuraminidase $\left(1 \mathrm{IU} / \mathrm{mL}\right.$ in $\left.\mathrm{PBS}, 30 \mathrm{~min}, 37^{\circ} \mathrm{C}\right)$, and the other was induced capacitation. The sperms were incubated with WGA-FITC at a final concentration of $30 \mu \mathrm{g} / \mathrm{mL}$ for $1 \mathrm{~h}$ at room temperature in a humid chamber. Then, the sperm was washed three times (at $800 \mathrm{~g}, 3 \mathrm{~min}$ at $37^{\circ} \mathrm{C}$ ) using PBS $(\mathrm{pH}$ 7.4) for removing the uncombined WGA-FITC, and the sperm pellets were resuspended in PBS for flow cytometer analysis. In addition, sperm was fixed for $2 \mathrm{~h}$ using $4 \%$ paraformaldehyde and then was placed on a clean coverslip and then air-dried to enable the cells to attach to the surface. They were then rehydrated with $\mathrm{PBS}$ for $10 \mathrm{~min}$ and incubated with a blocking solution containing $2 \%$ of BSA (Sigma) in PBS for $1 \mathrm{~h}$. After blocking, the sperm on cover slip was incubated with WGAFITC at a final concentration of $30 \mu \mathrm{g} / \mathrm{mL}$ for $1 \mathrm{~h}$ at room temperature in a humid chamber. The cover slip was washed three times in PBS for 5 min each time and then mounted with $50 \%$ glycerinum. Green fluorescence was observed under microscope (Nikon) at 400× magnification.

\section{Immunofluorescence}

Sperm cells were fixed in 4\% (w/v) paraformaldehyde (PBS/ PFA) for $30 \mathrm{~min}$ at room temperature. After washing three times with PBS, the sperm were resuspended in PBS and placed onto poly-L-lysine-coated coverslips (5- $\mu \mathrm{L}$ sperm suspension/ spot), and completely dried for $15 \mathrm{~min}$ at room temperature. The sperm were blocked in blocking buffer $3 \%$ BSA in PBS) at room temperature for $1 \mathrm{~h}$ and then incubated with primary antibodies AWN, PSP-1 (Du et al. 2016) and tyrosine phosphorylation antibody (Cat\# ab17302, Abcam) overnight at $4{ }^{\circ} \mathrm{C}$. The negative controls added PBS instead of primary antibodies. Then, slips were washed in PBS and incubated with FITC-conjugated secondary antibodies (Santa Cruz) for $2 \mathrm{~h}$. The slips were washed in PBS and counterstained with 4',6-diamidino-2-phenylindole (DAPI; Sigma) for identification of nuclei, and then examined using a fluorescent microscope.

\section{Protein enrichment using CMM-APBA beads}

Sperm pellets were collected after centrifugation at $800 \mathbf{g}$ for 5 min at $4^{\circ} \mathrm{C}$ and sperm lysis was acquired in RAPA buffer (RAPA:PMSF $=100: 1) .100 \mu \mathrm{L}$ sperm lysate $(1 \mu \mathrm{g} / \mu \mathrm{L})$ was diluted in $900 \mu \mathrm{L}$ PBS $(\mathrm{pH}$ 6.9). For removing nonspecific binding components, the CMM-APBA beads $(20 \mu \mathrm{L})$ were added into the supernatant and incubated on shaker at $4^{\circ} \mathrm{C}$ for $12 \mathrm{~h}$. CMM beads were used as negative control in the experiments. The supernatant was separated using the magnetic MagRack6. The beads were washed three times with PBS. Then, the proteins were eluted with $50 \mu \mathrm{L}$ of elution buffer $(\mathrm{pH} 10)$ added onto the beads and incubated on shaker at $4^{\circ} \mathrm{C}$ for $30 \mathrm{~min}$. The proteins were separated from beads using the magnetic MagRack6. This procedure was also repeated three times. The collected proteins were boiling with loading buffer for $10 \mathrm{~min}$.

\section{Western blot analysis}

Protein samples were boiled in $5 \times$ loading buffer for $10 \mathrm{~min}$ and separated by sodium dodecyl sulfate-polyacrylamide gel electrophoresis (SDS-PAGE). Subsequently, proteins were
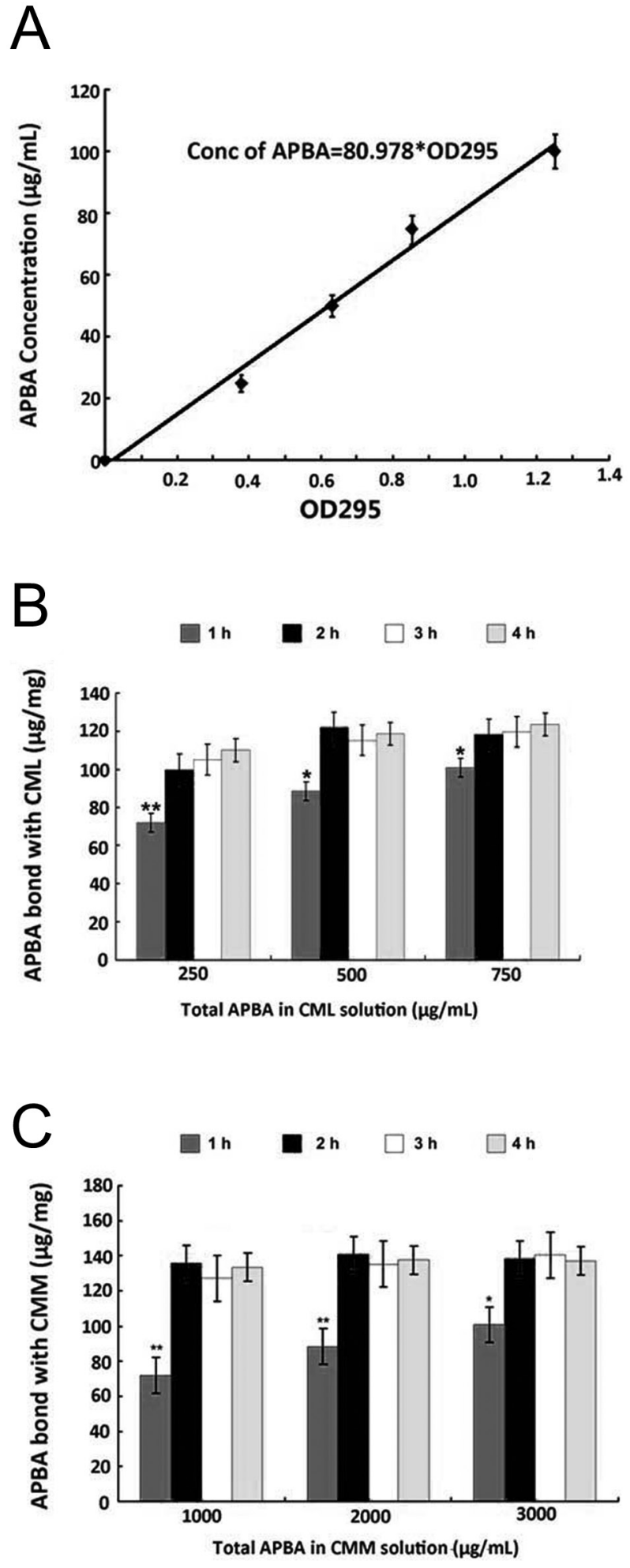

Figure 2 Analysis of the efficiency of APBA binding with CML or CMM. (A) The concentration of APBA in the solution, matching the OD value at absorption maximum wavelength at $259 \mathrm{~nm}$. (B) The efficiency of different concentrations of APBA in solution bound with CML at different reaction times. (C) The efficiency of different concentration APBA in solution bound with CMM at different reaction times. 
transferred to a polyvinylidene difluoride (PVDF) membrane. After blocking with 5\% (w/v) skim milk and 0.05\% (v/v) Tween 20 in Tris buffered saline (TBS; $20 \mathrm{mmol} / \mathrm{L}$ Tris, $500 \mathrm{mmol} / \mathrm{L}$ $\mathrm{NaCl} ; \mathrm{pH}$ 7.0), the blots were incubated with AWN, PSP-1 or tyrosine phosphorylation antibody at a dilution of 1:500. The membranes were washed with TBS containing $0.05 \%(\mathrm{v} / \mathrm{v})$ Tween 20 and subsequently incubated with HRP-conjugated anti-rabbit IgG antibody at a dilution of 1:2000. Specific antibody binding was detected using an ECL kit (Millipore).

Chemiluminescent signals were captured by digital camera or detection system (FluorChem ${ }^{\circledR} H \mathrm{D} 2$, Alpha, USA), and quantitative analysis was performed using NIH Image (Schneider et al. 2012). The relative level was calculated as ratio of density of target protein to that of beta1-tubulin.

\section{Statistical analysis}

All analyses were performed using Statistical Product and Service Solutions (SPSS 11.5 for windows; SPSS). All results were expressed as mean (s.D.) The mean values of the percentages of different kinds of patterns of sperm by CTC, CML-APBA and WGA-FITC staining were compared using Duncan's multiple range tests by ANOVA procedure, when the $F$ value was significant $(P<0.001)$. The correlation among the above-mentioned methods was evaluated by linear regression analysis.

\section{Results}

\section{Conjugation efficiency in the preparation processes of CML-APBA probe and CMM-APBA beads}

To measure the efficiency of APBA conjugated to CML or CMM, the concentration of APBA in the supernatant was detected by matching its absorption maximum at $295 \mathrm{~nm}$ to a predetermined standard curve in the same buffer (Fig. 2A), and the results were used to calculate the quantity of APBA bound to CML or CMM beads (Fig. 2B).
In a predetermined standard curve, the relationship between absorption spectra and concentration of APBA was linearity, and the optimal fitting equation was concentration of APBA $=80.978 \times$ value of OD259.

An excessive amount of APBA was added into the CML beads or CMM beads in the conjugation buffer. The APBA-CML (or CMM) obtained was ultrafiltrated twice to remove free APBA. The concentration of APBA in the filtrate was measured by matching its absorption maximum at $295 \mathrm{~nm}$ to a predetermined standard curve, and the results were used to calculate the content of APBA bound to beads. We found that the amount of APBA-bound CML or CMM was not affected by reaction time and the additional amount of APBA. Finally, the concentration of CML-APBA or CMM-APBA was $150 \pm 9.5 \mu \mathrm{g}$ APBA $/ \mathrm{mg} \mathrm{CML}$ and $143.8 \pm 5.6 \mu \mathrm{g} \mathrm{APBA} /$ mg CMM, respectively (Fig. $2 \mathrm{~B}$ and C).

\section{Capacitation assessment of boar sperm by CTC staining and protein tyrosine phosphorylation level analysis}

Increased level of tyrosine phosphorylation of sperm proteins is thought to be related to capacitation (Dorval et al. 2003, Bravo et al. 2005). In our study, the level of protein tyrosine phosphorylation of the Guanzhong Black boar sperm generally increased after capacitation (Fig. 3B).

Significant increase of spermatozoa with CTC staining fluorescent pattern B (Fig. 3A) and significant decrease of spermatozoa with CTC staining fluorescent pattern A (Fig. 3A) are a golden standard of capacitation (Ded et al. 2010). In our study, CTC staining pattern significantly changed in spermatozoa after capacitation in vitro. The rate of spermatozoa staining with staining pattern A was $84.5 \% \pm 1.95 \%$ before capacitation, but only $21.3 \% \pm 1.86 \%$ spermatozoa showed this pattern of staining after capacitation $(P<0.01)$. On the contrary,
A

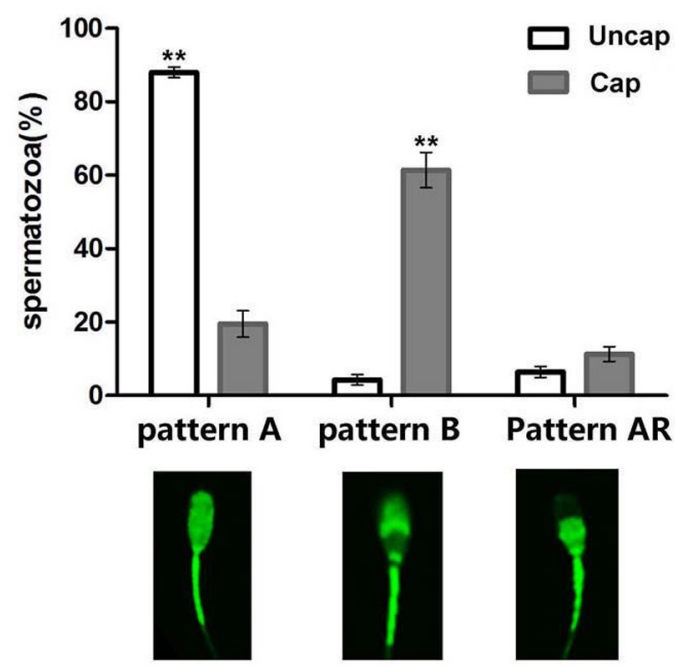

B

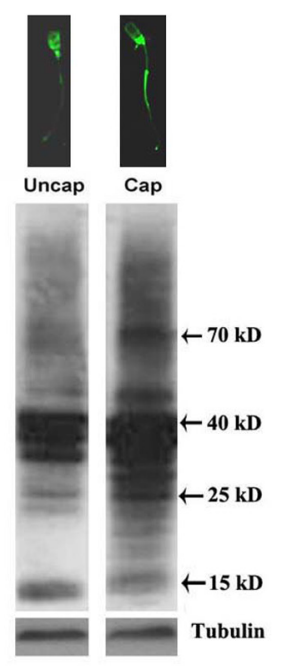

Figure 3 Identification of capacitation status of Guanzhong Black boar sperm. (A) Detection of capacitation of boar sperm by CTC assessment. Three patterns of chlortetracycline fluorescent staining were observed. Pattern A: Uncapacitated sperm; pattern B: Capacitated sperm; pattern AR: Acrosome-reacted sperm. (B) Change of the tyrosine phosphorylation during capacitaion. Uncap, uncapacitated sperm; Cap, capacitated sperm. The dual asterisk ${ }^{* *}$ ) represented the sperm number of the same fluorescence staining pattern was significant difference between after capacitation before capacitation $(P<0.001)$. 

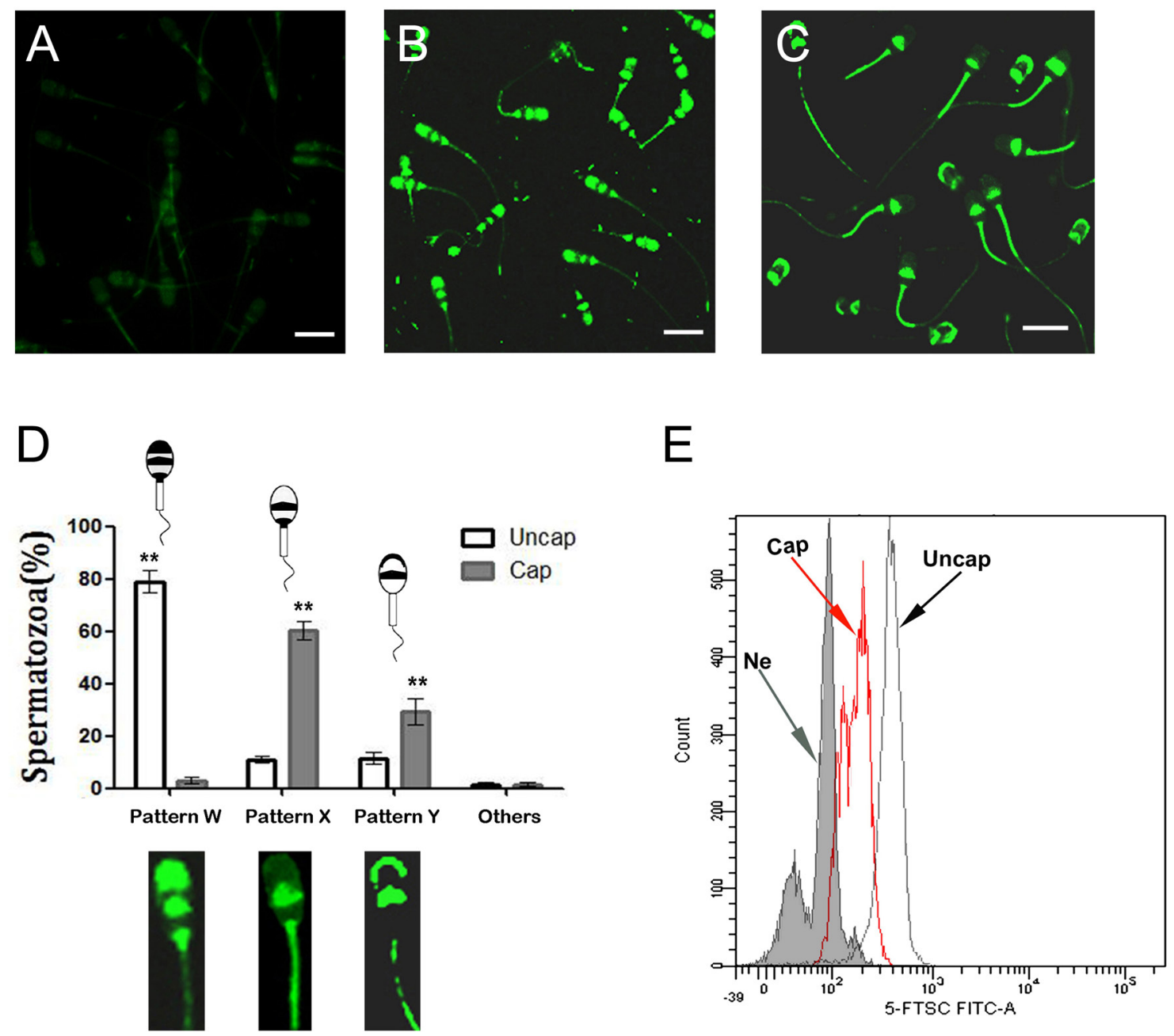

Figure 4 Location of sialic acid on sperm by using CML-APBA probe. (A) The uncapacitated sperm with neuraminidase treatment was stained by CML-APBA. (B) The uncapacitated sperm without neuraminidase treatment was stained by CML-APBA, pattern W was the fluorescence at the acrosomal region, equatorial segment, the posterior head of the sperm. (C) The capacitated sperm without neuraminidase treatment was stained by CML-APBA, there are two patterns: pattern $X$ was the fluorescenceat the equatorial segment and the head posterior of the sperm, and pattern $\mathrm{Y}$ was the fluorescence at acrosome region and the equatorial segment of the sperm. (D) Statistics of different patterns during capacitation by CML-APBA after capacitation before capacitation. (E) Fluorescence intensity of sperm binding CML-APBA was detected by flow cytometry. There's a lower fluorescence intensity of boar sperm after capacitation than before capacitation. Uncap, uncapacitated sperm; Cap, capacitated sperm; Ne, neuraminidase treatment; the dual asterisk $\left(^{* *}\right)$ represented the sperm number of the same fluorescence staining pattern was significant difference between after capacitation before capacitation $(P<0.001)$. Scale bar: $7 \mu \mathrm{m}$.

the percentage of spermatozoa with CTC staining pattern B was $4.30 \% \pm 0.16 \%$ before capacitation, while up to $61.9 \% \pm 1.95 \%$ spermatozoa had pattern $B$ of CTC staining after capacitation $(P<0.001)$. The results of protein tyrosine phosphorylation and CTC staining indicated that the protocol applied in this study was reliable for capacitation induction.

The assessment of sperm capacitation would be done for every sperm sample before use in the present study.

\section{Sialylation profiling on boar sperm surface with CML-APBA}

The uncapacitated and capacitated spermatozoa were incubated with CML-APBA probe in PBS with a $\mathrm{pH}$ value of 6.9 , and the spermatozoa were then washed and analyzed under fluorescence microscope (Fig. 4).

On the heads of uncapacitated spermatozoa, three green fluorescence sections could be detected: acrosomal region, equatorial segment and the head posterior (named as pattern W). At the principal segment and terminal of tail (Fig. 4B), the fluorescence signal could also be detected. After capacitation, spermatozoa were divided into two major groups according to the different locations of the green fluorescence signals on sperm surfaces: sperm with the fluorescence signals on equatorial segment and the head posterior (named as pattern X), and sperm with the fluorescence signals on acrosomal region and the equatorial segment (named as pattern Y). The green fluorescence signal was also observed at the principal 

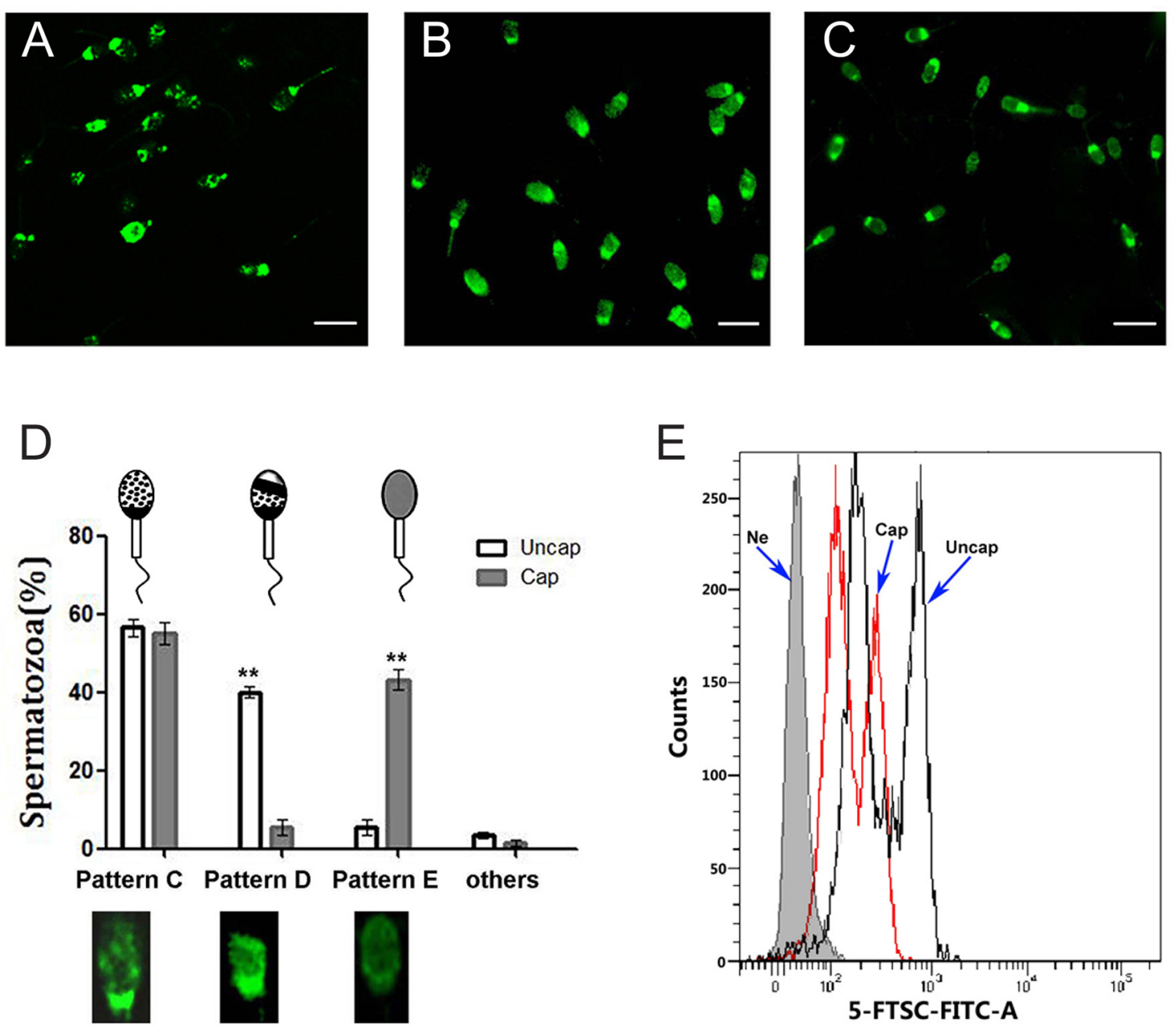

Figure 5 Location of sialic acid on sperm by FITC-WGA. (A) The uncapacitated sperm with neuraminidase treatment was stained by FITC-WGA. (B) The uncapacitation sperm without neuraminidase treatment was stained by FITC-WGA. (C) The capacitated sperm without neuraminidase treatment was stained by FITC-WGA. (D) Statistics of different patterns during capacitation by FITC-WGA. (E) Fluorescence intensity of sperm bounding FITC-WGA was tested by flow cytometry during capacitation. Uncap, uncapacitated sperm; Cap, capacitated sperm; Ne, neuraminidase treatment; the dual asterisk $\left.{ }^{* *}\right)$ represented the sperm number of the same fluorescence staining pattern was significant difference between after capacitation before capacitation $(P<0.001)$. Scale bar: $7 \mu \mathrm{m}$.

segment and terminal of tail (Fig. 4C). Furthermore, the results of flow cytometry analysis showed that the fluorescence signals on capacitated sperms significantly reduced (Fig. 4E), suggesting that the whole sialylation level decreased during capacitation.

In order to verify the recognition specificity of CMLAPBA probe for SA, the spermatozoa were treated with neuraminidase (also called sialidase) incubated with CML-APBA under the same condition. Little green fluorescence was detected on sperm under fluorescent microscope (Fig. 4A). Meanwhile, the green fluorescence signal could not be detected on sperm under fluorescent microscope when the sperm was incubated with $\mathrm{CML}$ at the same condition (data not shown). It was inferred that CML-APBA may be used as specific fluorescence probe to locate the SA on sperm and to monitor the changes of sialylation on boar spermatozoa.
Previous studies had shown that wheat germ agglutinin (WGA) has some affinity for SA (Nagata \& Burger 1974, Magargee et al. 1988). We also compared the difference of fluorescence location using the WGA-FITC by using the CML-APBA probe. Sperm of the Guanzhong Black boars was also incubated with WGA-FITC before and after capacitation. The results indicated that there were three fluorescence staining patterns in uncapacitated and capacitated sperm (Fig. 5B and C). More than half of the spermatozoa exhibited pattern $\mathrm{C}$, which is bright fluorescence at posterior of sperm head. However, there was no significant difference between the capacitated and uncapacitated spermatozoa. However, the rate of the pattern D, which was no fluorescence at acrosome of sperm head was higher in uncapacitated spermatozoa than in capacitated spermatozoa. In contrast with the pattern $D$, the percentage of the pattern $E$, which 
A

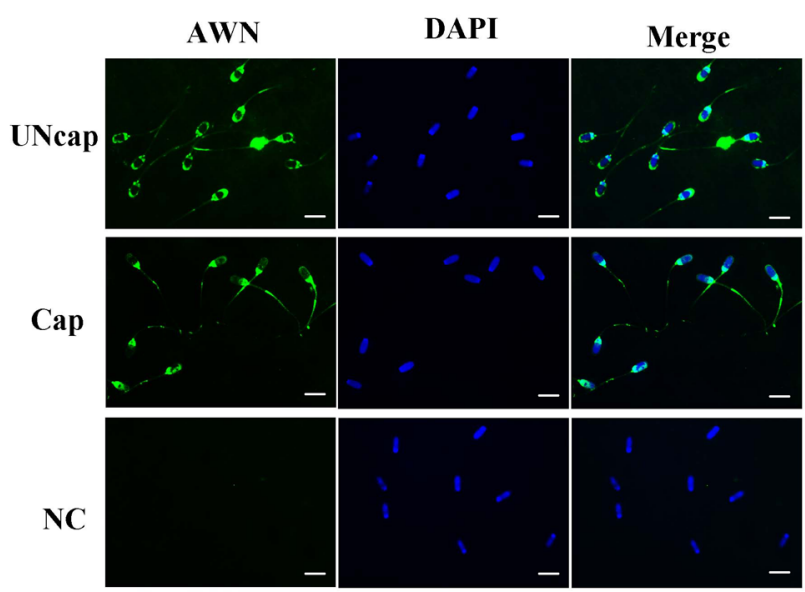

B

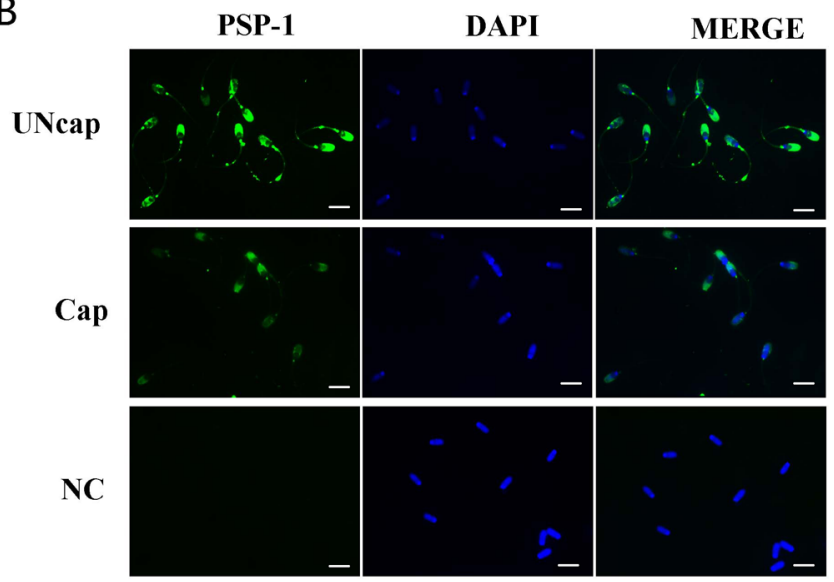

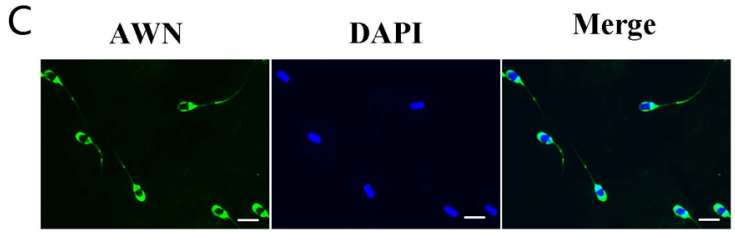
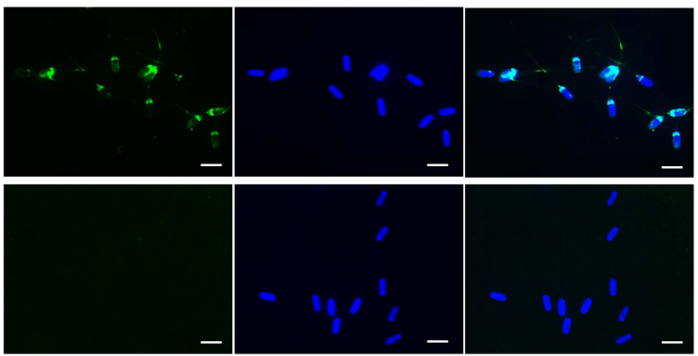

D
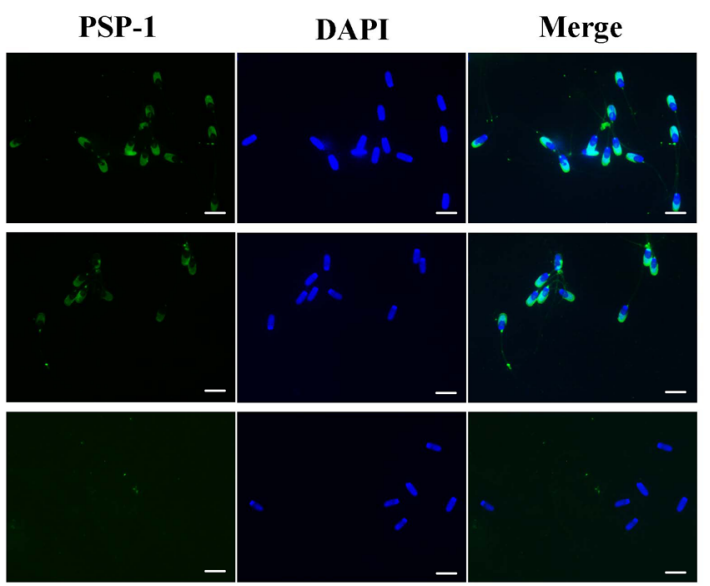

Figure 6 Immunofluorescence localization of AWN and PSP-1 on boar spermatozoa. (A) AWN was located on the acrosome region, the posterior head and the tail of uncapacitated sperm, and the fluorescence at the acrosome region was decreased after capacitation. (B) The localization of PSP-1 on sperm mainly focused on acrosome region, and it also decreased after capacitation. (C) Location of AWN on the sperm treated with neuraminidase. (D) Location of PSP-1 on the sperm treated with neuraminidase. Uncap, uncapacitated sperm; Cap, capacitated sperm. Scale bar: $7 \mu \mathrm{m}$.

was hypofluorescence on sperm head increased after capacitation (Fig. 5D). We also found that fluorescent spermatozoa could be divided into two groups, before capacitation as well as after capacitation, by flow cytometry analysis (Fig. 5E).

\section{Decapacitation factors $A W N$ and PSP-1 and their sialylation during boar sperm capacitation}

AWN and PSP-1, which are highly abundant proteins in boar seminal plasma, have been considered to be decapacitation factors (Dostálová et al. 1995, Varela et al. 1997). Immunofluorescence suggested that AWN and PSP-1 were located in the acrosomal region and tail of the uncapacitated sperm, and both their levels in acrosomal region decreased in in vitro capacitation (Fig. 6). On capacitated spermatozoa, AWN localized on the acrosome membrane and the head posterior, and PSP-1 appeared simultaneously on the acrosome membrane and the apical area of the principal piece of the sperm tail before capacitation in concurrence with previous reports (Sanz et al. 1992a, Manaskova \& Jonakova 2008, Villaverde et al. 2016). There is a partial overlap between the location of SA and AWN or PSP-1 (Fig. 6A and B). The localization of AWN or PSP-1 on sperm treated with neuraminidase did not seem to change (Fig. 6C and D). Western blot analysis was carried out for assessment of protein levels during capacitation (Fig. 7A).The levels of both PSP-1 and AWN decreased during capacitation. However, in the presence of neuraminidase, the level of PSP-1 $(P<0.01)$ on spermatozoa were less than that of control groups, but the level of AWN did not change, compared with that of control groups $(P>0.05)$. Furthermore, AWN or PSP-1 of capacitated spermatozoa could still be captured by CMM-APBA beads and detected 
A
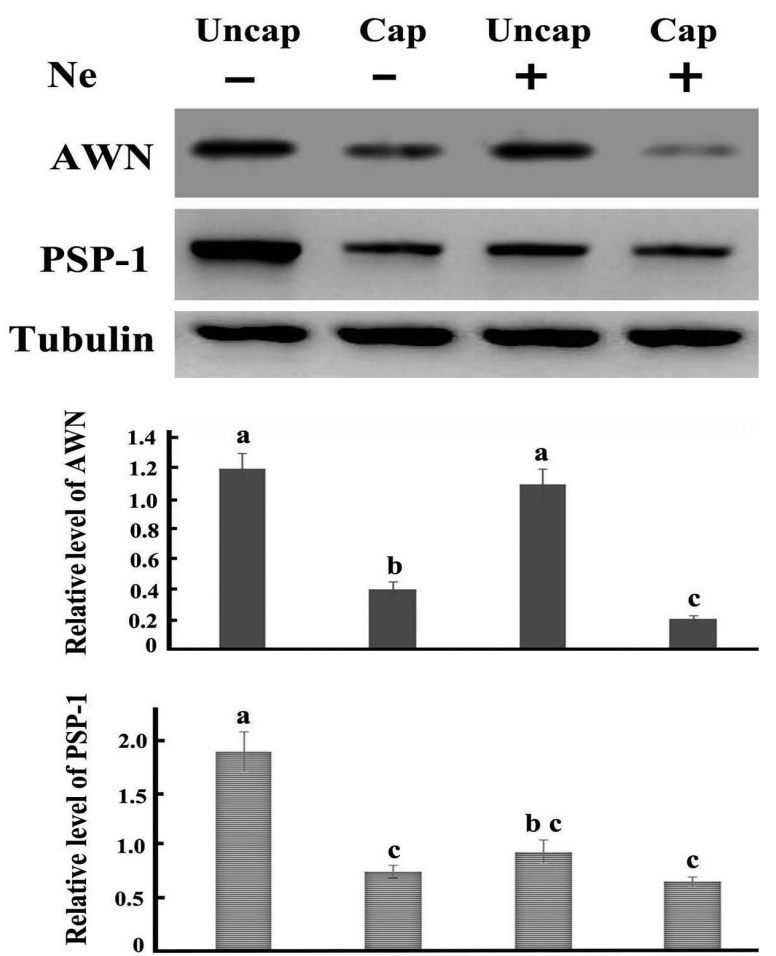

B

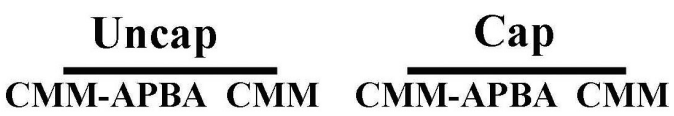

AWN

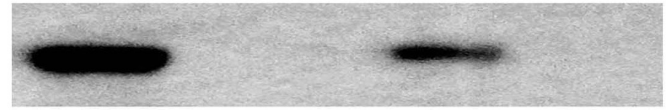

PSP-1

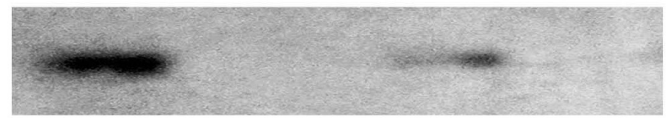

Figure 7 Identification of sialoglycoproteins AWN and PSP-1 and changes to them during capacitation. (A) Analysis of AWN and PSP-1 in boar sperm lysat by Western blot. Ne, neuraminidase; -, experiment groups without neuraminidase treatment; +, experiment groups with neuraminidase treatment. (B) Sialoglycoproteins were separated by CMM-APBA and analyzed by Western blot with anti-AWN or anti-PSP-1 antibodies respectively. Uncap, uncapacitated sperm; Cap, capacitated sperm. Different superscripts $(\mathrm{a}, \mathrm{b}$, and $\mathrm{c})$ mean significant differences $(P<0.05)$ among different treatments.

by Western blot analysis (Fig. 7B). AWN and PSP-1 were reduced after sperm capacitation. It not only further confirmed that AWN and PSP-1 are two sialylated proteins (Sanz et al. 1992b, Dostálová et al. 1995, Nimtz et al. 1999), but also suggested that the dissociation of AWN and PSP-1 from spermatozoa during capacitation may contribute to decreased sialylation on boar spermatozoa.

\section{Discussion}

Biological function of SA has been reported in various pathological and physiological processes. The invasion of some viruses and bacteria also depends on some specific SA patterns and aberrant sialylation is the feature of numerous of neurological, immune, cancer and congenital disorders of glycosylation (Yadav 2016). SA plays an important role in mammalian sperm undergoing epididymal maturation, capacitation and the acrosome reaction, and sperm-egg recognition (Pang et al. 2011, Ma et al. 2012, 2016).

There are some methods to research sialylation on mammalian sperm such as thiobarbituric acid assay (Diringer 1972), MBTH-method (Toowicharanont \& Chulavatnatol 1983), lectin probing (Jimenez et al. 2003), chromatograph (llinov et al. 1994) and so on. However, the confused results were reported using different methods by different research groups. Toowicharanont and coworkers reported that the content of SA of sperm decreased during sperm transferring from caput to cauda of epididymis by MBTH method (Toowicharanont \& Chulavatnatol 1983). But Magargee and coworkers thought that content of SA bound with sperm increased during sperm transition through epididymis by using fluorescence-labeled WGA lectin (Magargee et al. 1988). With chromatography, Ma and coworkers quantified SAs released by the presence of sperm sialidases ( $\mathrm{Ma}$ et al.2012), proving that sialylation decreases on mouse and human spermatozoa during capacitation. However, it has been accepted that lectin-bound fluorescences are capable to not only locate SA on cell surface, but also quantify the content of SA by flow cytometry, with a degree of non-specificity (Gomez-Torres et al. 2012, Villaverde et al. 2016).

Special affinity of boronic acid to SA at acidic environment was reported several decades ago and it has been extensively applied in cell labeling and sialylated biomolecule separation because of recent development of monolith materials (Lu et al. 2013). In our study, FITC latex beads chemically bound with 3-aminophenylboronic acid (CML-APBA) were prepared and used to localize the terminal SA residues on boar sperm surface as a fluorescent probe (Figs 1 and 2). Meanwhile, magnetic beads modified with APBA were prepared to enrich the sialylated proteins of sperm, which could be subsequently applied for quantity comparison of selected sialoglycoproteins by Western blot analysis.

Terminal SA residues on sperm surface could be located using CML-APBA with a high specificity. There were few fluorescent signals on spermatozoa after treatment with neuraminidase (sialidase), which suggested that the probe could specially bind with $\mathrm{SA}$ on the sperm membrane. We found that the green fluorescence signal appeared at three sections on sperm head before capacitation, while there were mainly two 
fluorescence groups of sperm after capacitation. The results showed that the distribution of SA by CML-APBA is different from WGA staining (Figs $4 \mathrm{~B}, \mathrm{C}$ and $5 \mathrm{~B}, \mathrm{C}$ ) (Magargee et al. 1988, Jimenez et al. 2003, Gomez-Torres et al. 2012). A reason is that WGA recognizes both sialic acid (NeuNAc) and $\mathrm{N}$-acetylglucosamine (GlcNAc) (Nagata \& Burger 1974), while boronic acid can favor binding with SA among common carbohydrates on cell surfaces at the physiological pH of 6.9 (Lu et al. 2013). Flow cytometry analysis revealed sialoglycoproteins of sperm surface decreased using CML-APBA as well as using WGA-FITC.

Interestingly, it has been reported that sialylation is abundant on zona pellucid (ZP) (Soupart \& Noyes 1964, Dunbar et al. 1980). Removal of SA residues from the ZP inhibits binding of spermatozoa to the ZP (Velasquez et al. 2007). On the contrary, ZP recognition was associated with the removal of SA from human sperm surface. It is reasonable to hypothesize that SAs of spermatozoa do not contribute to the binding of sperm to ZP, they act more like a protective coat to protect the sperm antigen before sperm-egg meeting. The decreased content of SAs may be related with the shedding of glycopeptide and sialoglycoproteins from spermatozoa (Srivastava et al. 1970, Focarelli et al. 1990, Ma et al. 2012).

We used the APBA magnetic beads-enriched sialoglycoproteins in sperm lysate and identified two sialylated proteins AWN and PSP-1 by Western blot analysis (Fig. 7B). Boar sperm adhesin AWN and PSP1 , as decapacitation factors, are both carbohydratebinding proteins peripherally attached to the external surface of spermatozoa (Calvete et al. 1995). Consistent with previous results, we demonstrated that $\mathrm{AWN}$ and PSP-1 are two sialylated proteins of boar sperm (Fig. 7B) (Sanz et al. 1992b, Nimtz et al. 1999). We found that the content of AWN of sperm treated with or without neuraminidase was decreased after capacitation. The content of PSP-1 of sperm treated with neuraminidase showed no significant change after capacitation, while level of PSP-1 on uncapacitated sperms decreased in the presence of neuraminidase (Fig. 7A). This suggests that dissociation of AWN or PSP-1 from spermatozoa depends on different mechanisms. Especially for PSP1 , presence of neuraminidase from spermatozoa during capacitation may result in the dissociation of PSP-1, which suggested that sperm neuraminidases not only desialylated sperm surface (Ma et al. 2012), but also help in the dissociation of decapacitation factors from boar spermatozoa.

\section{Conclusions}

A new method was established, which could mark SA on porcine sperm membrane and separate sialoglycoproteins of porcine spermatzoa at $\mathrm{pH} 6.9$ using carboxylate-modified latex beads (CML) or carboxylate-modified magnetic beads (CMM) modified with 3-aminophenylboronic acid (APBA), respectively. There are three green fluorescence sections on the sperm head: acrosomal region, equatorial segment and the head posterior before capacitation. After capacitation, there were two major groups of spermatozoa with different locations of sialylation. Flow cytometry analysis showed that level of sialylation on boar sperm membrane decreased after capacitation. Using CMMAPBA magnetic beads, we proved that AWN and PSP-1 were two sialoglycoproteins and the amounts of the two decapacitation factors were reduced after capacitation. It was inferred that dissociation of PSP-1 from sperm membrane may be related to desialylation carried out by sperm neuraminidases during capacitation.

\section{Declaration of interest}

All authors read and approved the final manuscript. The authors declare no competing financial interests.

\section{Funding}

This study was supported in part by the National Natural Science Foundation of China (Grant No. C170104-31172205), the National Swine Industry Technology System Foundation (CARS-36) and the Sci-Tech Project of Yangling Demonstration Zone (YDZ2017).

\section{Acknowledgments}

The authors are indebted to Prof. Wenxian Zeng and Prof. Gongshe Yang at Northwest A\&F University for comments and suggestion of the study.

\section{References}

Aitken RJ \& Nixon B 2013 Sperm capacitation: a distant landscape glimpsed but unexplored. Molecular Human Reproduction 19785. (https://doi.org/10.1093/molehr/gat067)

Bravo MM, Aparicio IM, Garcia-Herreros M, Gil MC, Pena FJ \& GarciaMarin LJ 2005 Changes in tyrosine phosphorylation associated with true capacitation and capacitation-like state in boar spermatozoa. Molecular Reproduction and Development 71 88-96. (https://doi.org/10.1002/ mrd.20286)

Calvete JJ, Mann K, Schäfer W, Raida M, Sanz L \& Töpferpetersen E 1995 Boar spermadhesin PSP-II: location of posttranslational modifications, heterodimer formation with PSP-I glycoforms and effect of dimerization on the ligand-binding capabilities of the subunits. FEBS Letters 365 179-182. (https://doi.org/10.1016/0014-5793(95)00452-F)

Cao J-T, Zhang P-H, Liu Y-M, Abdel-Halim E \& Zhu J-J 2015 Versatile microfluidic platform for the assessment of sialic acid expression on cancer cells using quantum dots with phenylboronic acid tags. ACS Applied Materials and Interfaces 7 14878-14884. (https://doi. org/10.1021/acsami.5b03519)

Ded L, Dostalova P, Dorosh A, Dvorakova-Hortova K \& Peknicova J 2010 Effect of estrogens on boar sperm capacitation in vitro. Reproductive Biology and Endocrinology 8 87. (https://doi.org/10.1186/1477-7827-887)

Diringer H 1972 The thiobarbituric acid assay of sialic acids in the presence of large amounts of lipid. Hoppe-Seyler's Zeitschrift Fur Physiologische Chemie 353 39-42. (https://doi.org/10.1515/bchm2.1972.353.1.39) 
Dorval V, Dufour M \& Leclerc P 2003 Role of protein tyrosine phosphorylation in the thapsigargin-induced intracellular $\mathrm{Ca} 2+$ store depletion during human sperm acrosome reaction. Molecular Human Reproduction 9 125-131. (https://doi.org/10.1093/molehr/gag017)

Dostálová Z, Calvete JJ, Sanz L \& Töpferpetersen E 1995 Boar spermadhesin AWN-1. Oligosaccharide and zona pellucida binding characteristics. European Journal of Biochemistry 230 329-336.

Du J, Shen J, Pan C, Pang Wn, Dao H \& Dong W 2016 Boar Seminal Plasma Exosomes Maintain Sperm Function by Infiltrating into the Sperm Membrane. Oncotarget 7 58832-58847. (https://doi.org/10.18632/ oncotarget.11315)

Dunbar BS, Wardrip NJ \& Hedrick JL 1980 Isolation, physicochemical properties, and macromolecular composition of zona pellucida from porcine oocytes. Biochemistry 19 356. (https://doi.org/10.1021/bi00543a017)

Estienne MJ, Harper AF \& Day JL 2007 Characteristics of sperm motility in boar semen diluted in different extenders and stored for seven days at $18^{\circ} \mathrm{C}$. Reproductive Biology 7 221-231.

Focarelli R, Rosati F \& Terrana B 1990 Sialyglycoconjugates release during in vitro capacitation of human spermatozoa. Journal of Andrology $\mathbf{1 1}$ 97-104.

Fraser LR 1998 Sperm capacitation and the acrosome reaction. Human Reproduction 13 (Supplement 1) 9-19. (https://doi.org/10.1093/ humrep/13.suppl_1.9)

Gomez-Torres MJ, Aviles M, Girela JL, Murcia V, Fernandez-Colom PJ, Romeu A \& De Juan J 2012 Characterization of the lectin binding pattern in human spermatozoa after swim-up selection. Histology and Histopathology 27 1621-1628. (https://doi.org/10.14670/HH-27.1621)

Holt WV 1980 Surface-bound sialic acid on ram and bull spermatozoa: deposition during epididymal transit and stability during washing. Biology of Reproduction 23 847-857. (https://doi.org/10.1095/ biolreprod23.4.847)

Huang L-L, Jin Y-J, Zhao D, Yu C, Hao J \& Xie H-Y 2014 A fast and biocompatible living virus labeling method based on sialic acidphenylboronic acid recognition system. Analytical and Bioanalytical Chemistry 406 2687-2693. (https://doi.org/10.1007/s00216-014-7651-9)

Ilinov PP, Deleva DD, Zaprianova ET \& Dimov SI 1994 A method for determination of lipid-bound sialic acid after chromatographic isolation of brain gangliosides. Journal of Liquid Chromatography and Related Technologies $17 \quad 2871-2879 . \quad$ (https://doi. org/10.1080/10826079408013505)

Jimenez I, Gonzalez-Marquez H, Ortiz R, Herrera JA, Garcii A, Betancourt M \& Fierro R 2003 Changes in the distribution of lectin receptors during capacitation and acrosome reaction in boar spermatozoa. Theriogenology 59 1171-1180. (https://doi.org/10.1016/ S0093-691X(02)01175-5)

Kumaresan A, Gonzalez R, Johannisson A \& Berqvist AS 2014 Dynamic quantification of intracellular calcium and protein tyrosine phosphorylation in cryopreserved boar spermatozoa during short-time incubation with oviductal fluid. Theriogenology 82 1145-1153. (https:// doi.org/10.1016/j.theriogenology.2014.07.029)

Kur-Kowalska K, Przybyt M, Ziolczyk P, Sowinski P \& Miller E 2014 Fluorescence properties of 3 -amino phenylboronic acid and its interaction with glucose and $\mathrm{ZnS}: \mathrm{Cu}$ quantum dots. Spectrochimica Acta Part A: Molecular and Biomolecular Spectroscopy 129 320-325. (https:// doi.org/10.1016/j.saa.2014.03.039)

Lee JH, Kim Y, Ha MY, Lee EK \& Choo J 2005 Immobilization of aminophenylboronic acid on magnetic beads for the direct determination of glycoproteins by matrix assisted laser desorption ionization mass spectrometry. Journal of the American Society for Mass Spectrometry 16 1456-1460. (https://doi.org/10.1016/j.jasms.2005.04.005)

Lin Z-A, Zheng J-N, Lin F, Zhang L, Cai Z \& Chen G-N 2011 Synthesis of magnetic nanoparticles with immobilized aminophenylboronic acid for selective capture of glycoproteins. Journal of Materials Chemistry $\mathbf{2 1}$ 518-524. (https://doi.org/10.1039/C0JM02300K)

Liu A, Peng S, Soo JC, Kuang M, Chen P \& Duan H 2011 Quantum dots with phenylboronic acid tags for specific labeling of sialic acids on living cells. Analytical Chemistry 83 1124-1130. (https://doi.org/10.1021/ ac1028853)

Lu Y, Bie Z, Liu Y \& Liu Z 2013 Fine-tuning the specificity of boronate affinity monoliths toward glycoproteins through $\mathrm{pH}$ manipulation. Analyst 138 290-298. (https://doi.org/10.1039/C2AN36048A)
Ma F, Wu D, Deng L, Secrest P, Zhao J, Varki N, Lindheim S \& Gagneux P 2012 Sialidases on mammalian sperm mediate deciduous sialylation during capacitation. Journal of Biological Chemistry 287 38073-38079. (https://doi.org/10.1074/jbc.M112.380584)

Ma X, Pan Q, Feng Y, Choudhury BP, Ma Q, Gagneux P \& Ma F 2016 Sialylation facilitates the maturation of mammalian sperm and affects its survival in female uterus. Biology of Reproduction 94 123. (https://doi. org/10.1095/biolreprod.115.137810)

Magargee SF, Kunze E \& Hammerstedt RH 1988 Changes in lectin-binding features of ram sperm surfaces associated with epididymal maturation and ejaculation. Biology of Reproduction 38 667-685. (https://doi. org/10.1095/biolreprod38.3.667)

Manaskova P \& Jonakova V 2008 Localization of porcine seminal plasma (PSP) proteins in the boar reproductive tract and spermatozoa. Journal of Reproductive Immunology 78 40-48. (https://doi.org/10.1016/j. jri.2007.10.001)

Nagata Y \& Burger MM 1974 Wheat germ agglutinin. Molecular characteristics and specificity for sugar binding. Journal of Biological Chemistry 249 3116-3122.

Nimtz M, Grabenhorst E, Conradt HS, Sanz L \& Calvete JJ 1999 Structural characterization of the oligosaccharide chains of native and crystallized boar seminal plasma spermadhesin PSP-I and PSP-II glycoforms. European Journal of Biochemistry 265 703-718. (https://doi. org/10.1046/j.1432-1327.1999.00766.x)

Ozerlat I 2011 Male factor infertility: mutation of sperm defensin causes subfertility. Nature Reviews Urology 8 474-474. (https://doi.org/10.1038/ nrurol.2011.115)

Pang PC, Chiu PC, Lee CL, Chang LY, Panico M, Morris HR, Haslam SM, Khoo KH, Clark GF \& Yeung WS 2011 Human sperm binding is mediated by the sialyl-Lewis(x) oligosaccharide on the zona pellucida. Science 333 1761-1764. (https://doi.org/10.1126/science.1207438)

Rohrer JS, Thayer J, Weitzhandler M \& Avdalovic N 1998 Analysis of the $\mathrm{N}$-acetylneuraminic acid and $\mathrm{N}$-glycolylneuraminic acid contents of glycoproteins by high-pH anion-exchange chromatography with pulsed amperometric detection (HPAEC/PAD). Glycobiology 8 35-43. (https:// doi.org/10.1093/glycob/8.1.35)

Sanz L, Calvete JJ, Jonáková V \& Töpferpetersen E 1992a Boar spermadhesins $\mathrm{AQN}-1$ and $\mathrm{AWN}$ are sperm-associated acrosin inhibitor acceptor proteins. FEBS Letters 300 63-66. (https://doi. org/10.1016/0014-5793(92)80164-C)

Sanz L, Calvete JJ, Mann K, Schäfer W, Schmid ER, Amselgruber W, Sinowatz F, Ehrhard M \& Töpfer-Petersen E $1992 b$ The complete primary structure of the spermadhesin $A W N$, a zona pellucida-binding protein isolated from boar spermatozoa. FEBS Letters 300 213-218. (https://doi. org/10.1016/0014-5793(92)80848-B)

Schauer R 1982 Chemistry, metabolism, and biological functions of sialic acids. Advances in Carbohydrate Chemistry and Biochemistry 40 131-234. (https://doi.org/10.1016/s0065-2318(08)60109-2)

Schneider CA, Rasband WS \& Eliceiri KW 2012 NIH image to Image): 25 years of image analysis. Nature Methods 9 671-675. (https://doi. org/10.1038/nmeth.2089)

Soupart, P \& Noyes RW 1964 Sialic acid as a component of the zona pellucida of the mammalian ovum. Journal of Reproduction and Fertility 8 251-253. (https://doi.org/10.1530/jrf.0.0080251)

Srivastava PN, Zaneveld LJ \& Williams WL 1970 Mammalian sperm acrosomal neuraminidases. Biochemical and Biophysical Research Communications 39 575-582. (https://doi.org/10.1016/0006291X(70)90242-1)

Tiralongo J \& Martinez-Duncker I 2013 Sialobiology: Structure, Biosynthesis and Function. Sialic Acid Glycoconjugates in Health and Disease. Bentham Science Publishers (https://doi.org/10.2174/9781608 0538651130101)

Tollner TL, Venners SA, Hollox EJ, Yudin AI, Liu X, Tang G, Xing H, Kays RJ, Lau T \& Overstreet JW 2011 A common mutation in the defensin DEFB126 causes impaired sperm function and subfertility. Science Translational Medicine 3 92ra65-92ra65. (https://doi.org/10.1126/ scitranslmed.3002289)

Toowicharanont P \& Chulavatnatol M 1983 Direct assay of bound sialic acids on rat spermatozoa from the caput and cauda epididymidis. Journal of Reproduction and Fertility 67 275-280. (https://doi. org/10.1530/jrf.0.0670275) 
Varela PF, Romero A, Sanz L, Romão MJ, Töpfer-Petersen E \& Calvete JJ 1997 The 2.4 A resolution crystal structure of boar seminal plasma PSP-I/PSP-II: a zona pellucida-binding glycoprotein heterodimer of the spermadhesin family built by a CUB domain architecture. Journal of Molecular Biology 274 635-649. (https://doi.org/10.1006/ jmbi.1997.1424)

Velasquez JG, Canovas S, Barajas P, Marcos J, Jimenez-Movilla M, Gallego RG, Ballesta J, Aviles M \& Coy P 2007 Role of sialic acid in bovine sperm-zona pellucida binding. Molecular Reproduction and Development 74 617-628. (https://doi.org/10.1002/mrd.20619)

Villaverde AI, Hetherington L \& Baker MA 2016 Quantitative glycopeptide changes in rat sperm during epididymal transit. Biology of Reproduction 94 91. (https://doi.org/10.1095/biolreprod.115.134114)

Wang WH, Abeydeera LR, Fraser LR \& Niwa K 1995 Functional analysis using chlortetracycline fluorescence and in vitro fertilization of frozenthawed ejaculated boar spermatozoa incubated in a protein-free chemically defined medium. Journal of Reproduction and Fertility 104 305. (https://doi.org/10.1530/jrf.0.1040305)
Wang X, Xia N \& Liu L 2013 Boronic acid-based approach for separation and immobilization of glycoproteins and its application in sensing. International Journal of Molecular Sciences 14 20890-20912. (https:// doi.org/10.3390/ijms141020890)

Yadav R 2016 Exploring the Structural and Functional Aspects of Sialic acid Mimetics. Pune: IISER.

Young LG, Gould KG \& Hinton BT 1986 Lectin binding sites on the plasma membrane of epididymal and ejaculated chimpanzee sperm. Gamete Research 14 75-87. (https://doi.org/10.1002/mrd.1120140109)

Received 19 June 2017

First decision 18 July 2017

Revised manuscript received 29 September 2017

Accepted 17 October 2017 